\title{
Intersectin: the crossroad between vesicle exocytosis and endocytosis
}

\section{Olga Gubar 1,2,3, Dmytro Morderer 1,2, Lyudmila Tsyba², Pauline Croisé ${ }^{3}$, Sébastien Houy ${ }^{3}$, Stéphane Ory³, Stéphane Gasman ${ }^{3}$ and Alla Rynditch ${ }^{1,2}$ *}

\author{
1 State Key Laboratory of Molecular and Cellular Biology, Institute of Molecular Biology and Genetics, Kyiv, Ukraine \\ ${ }^{2}$ Department of Functional Genomics, Institute of Molecular Biology and Genetics, Kyiv, Ukraine \\ ${ }^{3}$ Institut des Neurosciences Cellulaires et Intégratives, Centre National de la Recherche Scientifique-Unité Propre de Recherche 3212, Université de Strasbourg, \\ Strasbourg, France
}

\section{Edited by:}

Rafael Vazquez-Martinez, University

of Cordoba, Spain

Reviewed by:

Pedro A. Jose, Georgetown

University, USA

Sanda Predescu, Rush University

Medical Center, USA

*Correspondence:

Alla Rynditch, Department of

Functional Genomics, Institute of

Molecular Biology and Genetics, 150

Zabolotnogo Street, 03680 Kyiv-143,

Ukraine

e-mail: rynditch@imbg.org.ua
Intersectins (ITSNs) are a family of highly conserved proteins with orthologs from nematodes to mammals. In vertebrates, ITSNs are encoded by two genes (itsn 1 and itsn2), which act as scaffolds that were initially discovered as proteins involved in endocytosis. Further investigation demonstrated that ITSN1 is also implicated in several other processes including regulated exocytosis, thereby suggesting a role for ITSN1 in the coupling between exocytosis and endocytosis in excitatory cells. Despite a high degree of conservation amongst orthologs, ITSN function is not so well preserved as they have acquired new properties during evolution. In this review, we will discuss the role of ITSN1 and its orthologs in exo- and endocytosis, in particular in neurons and neuroendocrine cells.

Keywords: intersectin, exocytosis, endocytosis, neurons, neuroendocrine cells, Cdc42, scaffold

\section{ITSN FAMILY OF SCAFFOLD PROTEINS}

Intersectins (ITSNs) are multifunctional scaffold proteins implicated in several cellular mechanisms, including membrane trafficking (clathrin- and caveolin-mediated endocytosis, secretagogue-evoked exocytosis) and receptor-dependent signaling (Ras-MAPK and Rho GTPase regulation, EGF receptor ubiquitylation) to name a few [extensively reviewed in (1-3)]. Based on the high involvement of ITSNs in membrane trafficking, this mini review will focus on the role of these proteins in exocytosis and endocytosis in neurosecretory cells such as neurons and neuroendocrine cells, and will discuss how ITSNs could be key players in coupling exocytosis to endocytosis.

Intersectin is highly conserved in all metazoans examined so far and ITSN orthologs have been found in nematodes (Caenorhabditis elegans) (4), arthropods (Drosophila melanogaster Dap160 - Dynamin-associated protein $160 \mathrm{kDa})(5)$, fish (Danio rerio) (6), amphibians (Xenopus laevis) (7), and mammals (Mus musculus, Rattus norvegicus, Homo sapiens) (8-10) (Figure 1). ITSN is encoded by one gene in invertebrates. Its molecular organization consists of two N-terminal EH (Eps15 homology) domains followed by a coiled-coil region and four or five SH3 (Src homology 3) domains (5). In vertebrates, two genes encode ITSN proteins (ITSN1 and ITSN2). In addition, ITSNs exist in two main isoforms generated by alternative splicing: a short form (ITSN-S) that harbors the same domain organization as invertebrate ITSN and is ubiquitously expressed and a long form (ITSN-L) which has three additional domains in its C-terminal part [tandem of $\mathrm{Dbl}(\mathrm{DH})$ and pleckstrin homology (PH) domains and a C2 domain] (10). This extension has guanine nucleotide exchange factor (GEF) properties for Cdc42 (11), a small GTPase of the Rho family. The ITSN1-L is enriched in neurons $(10,12)$.

\section{ITSN1 IN NEURONAL ENDOCYTOSIS}

Multimodular ITSN1 has been shown to interact with numerous endocytic proteins, including dynamin, AP2 (adaptor protein 2), proteins from the Epsin family, and the synaptojanin phosphatase; its function in the control of endocytosis has been described in different cell types and organisms [reviewed in (1, 2)]. The role of ITSN1 in endocytosis in neurons was further demonstrated in functional assays performed in various model organisms. C. elegans containing deletions in the itsn-1 gene were hypersensitive to the acetylcholine esterase inhibitor aldicarb that is widely used for unmasking neurotransmission defects. It causes rapid hypercontraction and eventual death of wild-type worms. This itsn-1-null aldicarb-hypersensitive phenotype could be rescued by expressing ITSN-1 in neurons, but not in muscles, indicating a pre-synaptic dysfunction in mutant animals (4). Another study revealed a decrease in the number of vesicles in neuromuscular junctions (NMJ) of itsn-1-null worms, implying that ITSN-1 was required for synaptic vesicle (SV) recycling (13). In both cases, the mutant worms were viable and exhibited no changes in growth and locomotor activity, implying that the nervous system functioned normally. This suggests that ITSN-1 is not essential for neuronal endocytosis in nematodes, but rather plays a regulatory role increasing the efficiency of this process. Worm ITSN-1 has been shown to form a complex with the Eps15 ortholog EHS-1 (13) and a mutation in the ehs-1 gene also leads to SV depletion. However, ehs-1/itsn-1 double mutants develop a phenotype similar to that of single 


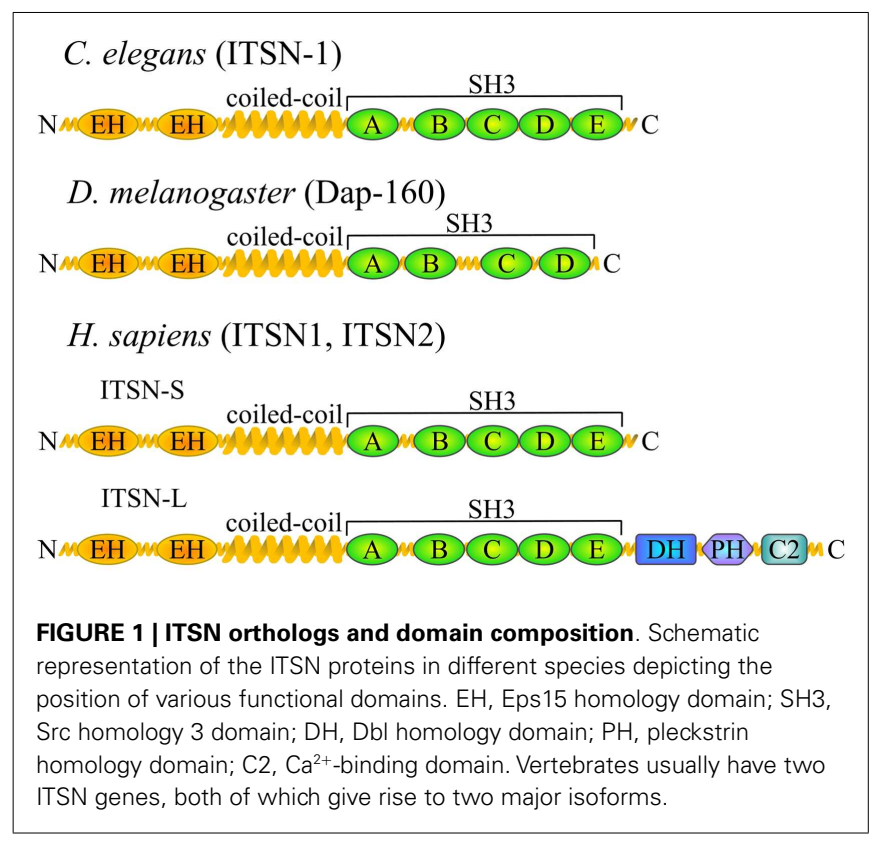

mutants, supporting the idea that these proteins act in the same process $(13,14)$. Intriguingly, itsn-1 and ehs-1 mutants have opposite sensitivities to aldicarb, indicating that they have different effects on cholinergic neurotransmission, most likely at the pre-synaptic level (4). Finally, in addition to pre-synaptic functions, ITSN-1 is required for internalization of the GLR-1 (glutamate receptor 1 ) subunit of the $\alpha$-amino-3-hydroxy-5-methyl4-isoxazolepropionic acid-type glutamate (AMPA) receptors in the post-synaptic terminals of nematode command interneurons (15). Thus ITSN-1 seems to be important for both pre- and post-synaptic endocytosis in worms.

In contrast to nematodes, the loss-of-function mutation of the ITSN ortholog Dap160 in Drosophila is lethal at late larval stages. Functional studies on the NMJ of mutant flies did not reveal significant changes in stimuli-evoked SV exocytosis; however, prolonged stimulation led to a severe decline in the excitatory post-synaptic potential (EPSP), indicating impairment of compensatory endocytosis $(16,17)$. As in nematodes, the number of vesicles in the active zones decreased in the NMJ of mutant Drosophila (16). In agreement with a function of ITSN in tethering endocytic proteins to the sites of endocytosis, the loss of Dap160 leads to mislocalization of dynamin, endophilin, and synaptojanin, resulting in decreased levels of these proteins in periactive synaptic zones $(16,17)$. Interestingly, overexpression of Dap160 also leads to a decrease in the level of synaptojanin, a $5^{\prime}$-phosphoinositide phosphatase involved in late stages of endocytosis, in NMJ synaptic terminals (18), indicating that Dap160/ITSN1 functions as a scaffold in endocytosis and is required to maintain endocytic proteins in synaptic terminals at levels necessary for efficient retrieval of SVs.

Studies on lamprey giant reticulospinal synapse have confirmed the data obtained in invertebrates concerning the involvement of ITSN1 in SV endocytosis. Perturbation of ITSN1 function by injecting either anti-ITSN1 antibodies or the SH3C domain into the pre-synaptic terminal resulted in an accumulation of clathrin-coated pits and a reduction in SV number, suggesting a defect in compensatory endocytosis $(19,20)$. It has also been shown that ITSN1 binds the AP2 clathrin adaptor both in lamprey and mammalian brain, suggesting that ITSN1 participates in the early steps of clathrin-mediated SV endocytosis. Moreover, such an interaction prevents ITSN1 from associating with synaptojanin 1 (20). These findings imply that ITSN1 temporally regulates SV endocytosis.

The data regarding the role of ITSN1 in SV endocytosis in mammals remain contradictory. ITSN1 along with Eps15 have been shown to form a complex with FCHo (Fer/Cip4 homology domain-only) proteins, that promotes the initiation of clathrincoated pit formation - the step required for clathrin-mediated endocytosis of different cargo in various cell types, including SV endocytosis in neurons (21). ITSN1 isoforms have also been identified as members of the synaptotagmin I-associated endocytic complex in the synaptosomal fraction of rat brain, and were subsequently shown to co-localize with other members of this complex in the pre-synaptic transmitter-release face of the giant calyx-type synapse of the chick ciliary ganglion (22). Knockout of ITSN1 in mice slows down SV endocytosis in neurons, although the animals are viable (23). However, another group found that SV endocytosis remains normal following ITSN1 knock-down in rat primary hippocampal neurons. This was demonstrated by the absence of a decrease in the uptake of FM4-64 membrane dye after $\mathrm{KCl}$ stimulation (24). Moreover, using capacitance measurements of plasma membrane in knockout mouse calyx of Held synapse, Sakaba and co-workers have recently shown that ITSN1 is not essential for endocytic SV recovery after pulse stimulation (25). Furthermore, several authors have reported that ITSN1 is mostly localized post-synaptically in hippocampal neurons, (24, 26), although some data indicating a pre-synaptic localization in mammalian synapses also exist $(20,25)$. Finally, recent electrophysiological studies did not reveal abnormalities in synaptic transmission either in ITSN1 knockout mice or in ITSN1/ITSN2 double knockout mice using a wide range of stimulation protocols, indicating that under the conditions tested SV recycling is not perturbed (27). Therefore it seems possible that in mammals, in contrast to invertebrates, ITSN1 acts predominantly postsynaptically; however, we cannot totally exclude it is an accessory protein in SV recycling.

Thus the role of ITSN in neuronal endocytosis apparently has not been conserved during evolution. In some groups of organisms (e.g., arthropods) ITSN is indispensable for normal SV recycling, whereas in higher evolved organisms it has become non-essential probably due to the development of alternative or compensatory mechanisms. In lower organisms, these functions are fulfilled by the short isoform, whereas in higher organisms (e.g., vertebrates) ITSN1-L is the likely candidate as it is the main isoform in neurons.

\section{ITSN1 FUNCTION IN EXOCYTOSIS IN NEUROENDOCRINE CELLS}

There is much less evidence about the implication of ITSN in exocytosis. One of the first studies reported the interaction of ITSN1 with t-SNAREs [target Soluble NSF Attachment Protein (SNAP) Receptor] SNAP-23 and SNAP-25, but no functional consequence was demonstrated (9). A new specific function of ITSN1-L as GEF 
for Cdc42 in exocytosis was described in neuroendocrine cells. In regulated exocytosis, remodeling of the dense actin cortical network is an important step that is controlled by small GTPases. The key players in this process are the Rho family GTPases [reviewed in $(28,29)]$. In the PC12 rat pheochromocytoma secretory model, Cdc42 was shown to be activated near the plasma membrane during exocytosis, where it recruits Neural Wiskott-Aldrich syndrome protein (N-WASP) and induces actin polymerization (30). ITSN1-L appeared to be an ideal candidate for Cdc42 activation at docking sites for secretory granules, because it is a specific GEF for Cdc42 and at the same time binds to its effector N-WASP (11). This leads to local polymerization of actin, thereby facilitating exocytosis. ITSN1-L was observed to co-localize with exocytic sites in PC12 and primary bovine chromaffin cells (31). Moreover, silencing of ITSN1 (as well as of Cdc42) significantly inhibits regulated exocytosis in PC12 cells, whereas overexpression of the C-terminal part of ITSN1-L (DH-PH-C2 domains) promotes exocytosis and peripheral actin polymerization in neuroendocrine cells $(31,32)$. These results were confirmed in ITSN1-null mice where exocytosis is also reduced in chromaffin cells (23). Finally, ITSN1 was very recently shown to regulate the replenishment of the fast-releasing SV pool in mouse calyx of Held synapse, possibly together with dynamin 1 and as a GEF for Cdc42 (25). Thus ITSN1-L displays novel properties in membrane trafficking distinct from those of ITSN1-S that were probably acquired during evolution.

\section{ITSN AS A PLAUSIBLE LINIK BETWEEN EXO- AND ENDOCYTOSIS}

Since ITSN1, especially the isoform ITSN1-L, is strongly implicated in both endo- and exocytosis it is an ideal protein for the coupling of these processes. Regulated exocytosis is always followed by compensatory endocytosis, which is also $\mathrm{Ca}^{2+}$-dependent and is stimulated together with exocytosis (33). Thus the presence of a calcium-binding C2 domain in ITSN-L may be a key for its implication in both exo- and endocytosis. Moreover, in studies on the chick ciliary ganglion ITSN1-S and ITSN1-L have been reported to be associated with the CaV2.2 calcium channels at transmitter-release sites (34).

In neurons, the active zone is highly structured to provide maximum secretory efficiency. The protein matrix, which consists of the cytoskeleton and scaffold proteins, ensures efficient SV docking, whereas electron-dense projections from this matrix serve to tether and maintain the ready-to-use SV pool (35). It is surrounded by a periactive zone that serves for SV recycling by endocytosis. A tight connection and coordination of SV release and recycling is provided by scaffolds [reviewed in (36)]. ITSN1 is a perfect candidate to fulfill this role. During synaptic activity, Dap160 has recently been shown to shuttle between active and periactive zones, ensuring the delivery of dynamin to the periactive zone for efficient bulk membrane retrieval (37). In the lamprey giant reticulospinal synapse, ITSN1 is also localized in the active zone of resting synapses, but re-localizes with its major
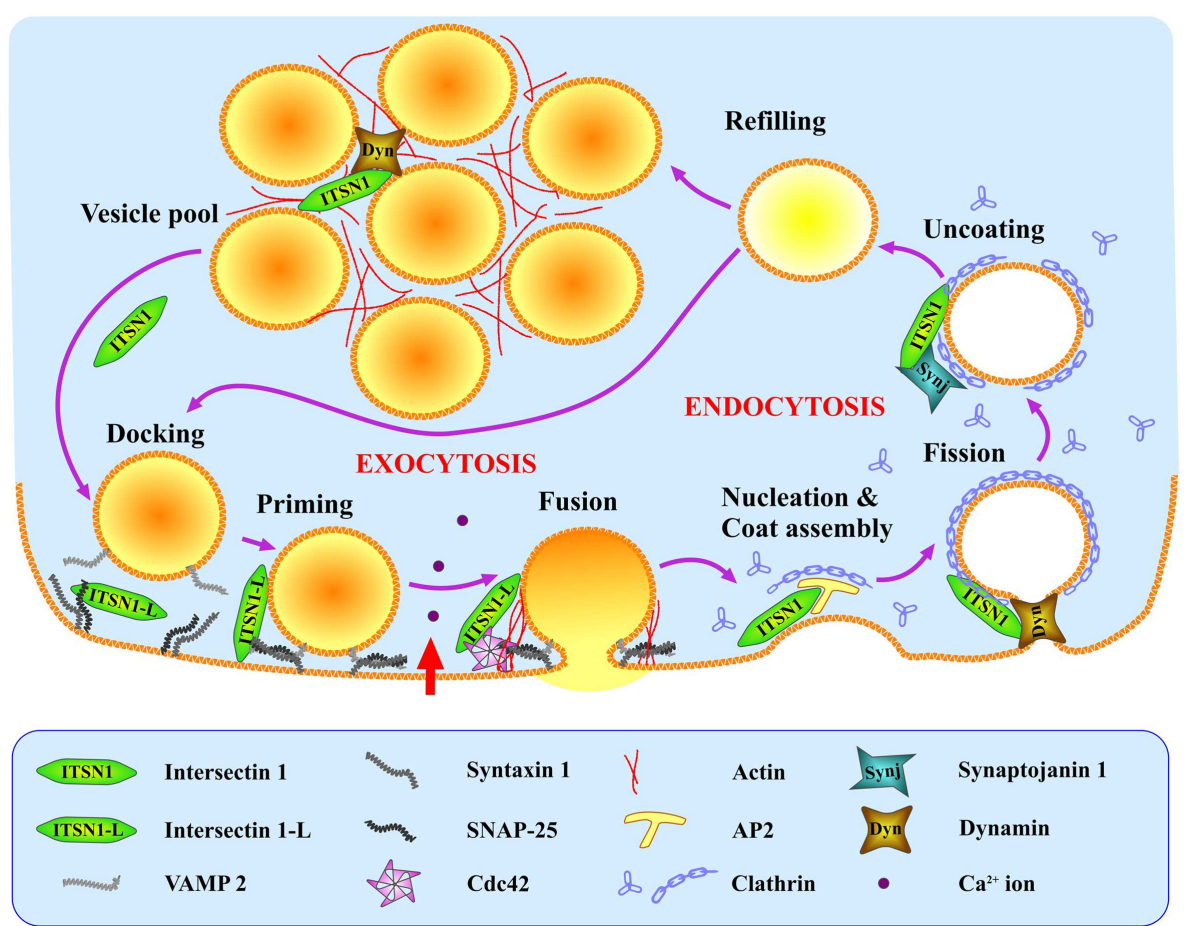

FIGURE 2 | Hypothetical model of ITSN1 coupling exo- and endocytosis in neuroendocrine cells. SNAP-25 recruits ITSN1-L to the SNARE complex (VAMP2, SNAP-25, syntaxin 1) at the exocytic sites where ITSN1-L facilitates regulated exocytosis by activating $\mathrm{Cdc} 42$. Immediately after vesicle fusion and hormone release, ITSN1 promotes clathrin-mediated endocytosis of the granule membrane via AP2, and facilitates later stages of endocytosis together with dynamin and synaptojanin. ITSN1 then stays in the vesicle cluster (probably with dynamin) to be recruited back to the membrane for the next release cycle. ITSN1, total ITSN1 (both short and long isoforms); ITSN1-L, long isoform of ITSN1. 
binding partner dynamin to the periactive zone upon stimulation (19). These results support the idea that ITSN1 acts as a scaffold coupling vesicle fusion and internalization events.

Although to date there is currently no direct evidence that ITSN1 plays a role in compensatory endocytosis in neuroendocrine cells, such data do exist for other cell types (including neurons). Based on these data and the presence of ITSN1 partners in neuroendocrine cells, we propose the following hypothetical model of ITSN1 functioning in exo- and endocytosis in these cells (Figure 2). ITSN1 may function in both the release and retrieval of the dense-core granules. SNAP-25 could recruit ITSN1-L to exocytic sites where the latter activates $\mathrm{Cdc} 42$ thereby inducing actin polymerization and facilitating the late stages of secretion $(9,31,32)$. After release, ITSN1-L remaining on the vesicle membrane could directly promote assembly of the endocytic complex, because the vesicle membrane has been shown to be preserved as an entity during the compensatory endocytosis in neuroendocrine

\section{REFERENCES}

1. O’Bryan JP. Intersecting pathways in cell biology. Sci Signal (2010) 3(152):re10. doi:10.1126/scisignal. 3152re10

2. Tsyba L, Nikolaienko O, Dergai O, Dergai M, Novokhatska O, Skrypkina I, et al. Intersectin multidomain adaptor proteins: regulation of functional diversity. Gene (2011) 473(2):67-75. doi:10.1016/j. gene.2010.11.016

3. Hunter MP, Russo A, O’Bryan JP. Emerging roles for intersectin (ITSN) in regulating signaling and disease pathways. Int $\mathrm{J} \mathrm{Mol} \mathrm{Sci}$ (2013) 14(4):7829-52. doi:10.3390/ ijms14047829

4. Rose S, Malabarba MG, Krag C, Schultz A, Tsushima H, Di Fiore PP, et al. Caenorhabditis elegans intersectin: a synaptic protein regulating neurotransmission. Mol Biol Cell (2007) 18(12):5091-9. doi:10.1091/ mbc.E07-05-0460

5. Roos J, Kelly RB. Dap160, a neuralspecific Eps15 homology and multiple SH3 domain-containing protein that interacts with Drosophila dynamin. J Biol Chem (1998) 273(30):19108-19. doi:10.1074/jbc. 273.30.19108

6. Dergai M, Tsyba L, Dergai O, Zlatskii I, Skrypkina I, Kovalenko $\mathrm{V}$, et al. Microexon-based regulation of ITSN1 and Src SH3 domains specificity relies on introduction of charged amino acids into the interaction interface. Biochem Biophys Res Commun (2010) 399(2):307-12. doi:10.1016/j.bbrc.2010.07.080

7. Yamabhai M, Hoffman NG, Hardison NL, McPherson PS, Castagnoli L, Cesareni G, et al. Intersectin, a novel adaptor protein with two Eps15 homology and five Src homology 3 domains. J Biol
Chem (1998) 273(47):31401-7. doi: 10.1074/jbc.273.47.31401

8. Sengar AS, Wang W, Bishay J, Cohen $\mathrm{S}$, Egan SE. The EH and SH3 domain Ese proteins regulate endocytosis by linking to dynamin and Eps15. EMBO J (1999) 18(5):1159-71. doi: 10.1093/emboj/18.5.1159

9. Okamoto M, Schoch S, Südhof TC. EHSH1/intersectin, a protein that contains $\mathrm{EH}$ and $\mathrm{SH} 3$ domains and binds to dynamin and SNAP-25. A protein connection between exocytosis and endocytosis? J Biol Chem (1999) 274(26):18446-54. doi:10. $1074 /$ jbc.274.26.18446

10. Guipponi M, Scott HS, Hattori M, Ishii K, Sakaki Y, Antonarakis SE. Genomic structure, sequence, and refined mapping of the human intersectin gene (ITSN), which encompasses $250 \mathrm{~kb}$ on chromosome 21q22.1->q22.2. Cytogenet Cell Genet (1998) 83(3-4):218-20. doi:10.1159/000015182

11. Hussain NK, Jenna S, Glogauer M, Quinn CC, Wasiak S, Guipponi $\mathrm{M}$, et al. Endocytic protein intersectin-1 regulates actin assembly via Cdc42 and N-WASP. Nat Cell Biol (2001) 3(10):927-32. doi: 10.1038/ncb1001-927

12. Hussain NK, Yamabhai M, Ramjaun AR, Guy AM, Baranes D, O'Bryan JP, et al. Splice variants of intersectin are components of the endocytic machinery in neurons and nonneuronal cells. J Biol Chem (1999) 274(22):15671-7. doi: 10.1074/jbc.274.22.15671

13. Wang W, Bouhours M, Gracheva EO, Liao EH, Xu K, Sengar AS, et al. ITSN-1 controls vesicle recycling at the neuromuscular junction and functions in parallel with DAB-1. Traffic (2008) 9(5):742-54. doi:10. 1111/j.1600-0854.2008.00712.x cells (38). Otherwise, ITSN1 could be recruited de novo and induce clathrin coat assembly via AP2 (20). Then ITSN1, in turn, can recruit dynamin to provoke vesicle scission $(8,9)$. After vesicle dissociation from the membrane, AP2 could be replaced by synaptojanin 1 , which takes part in the uncoating and dephosphorylation of $\mathrm{PIP}_{2}$ (phosphatidylinositol 4,5-bisphosphate) (20). The vesicle is then refilled and can be tethered ready for another cycle of exocytosis or added to the releasable vesicle pool. ITSN1 may accompany the vesicle throughout this cycle and remain in the releasable pool as it does in neurons (19). Thus ITSN1 could be a multipurpose player providing the crossroad between exo- and endocytosis in neurosecretory cells and an important regulator of these processes.

\section{ACKNOWLEDGMENTS}

We thank Dr. A.-L. Haenni and Dr. N. Grant for helpful discussions and comments on the manuscript.

14. Salcini AE, Hilliard MA, Croce A, Arbucci S, Luzzi P, Tacchetti C, et al. The Eps15 C. elegans homologue EHS-1 is implicated in synaptic vesicle recycling. Nat Cell Biol (2001) 3(8):755-60. doi:10.1038/35087075

15. Glodowski DR, Chen CC, Schaefer H, Grant BD, Rongo C. RAB-10 regulates glutamate receptor recycling in a cholesterol-dependent endocytosis pathway. Mol Biol Cell (2007) 18(11):4387-96. doi:10.1091/mbc. E07-05-0486

16. Koh TW, Verstreken P, Bellen HJ. Dap160/intersectin acts as a stabilizing scaffold required for synaptic development and vesicle endocytosis. Neuron (2004) 43(2):193-205. doi:10.1016/j.neuron.2004.06.029

17. Marie B, Sweeney ST, Poskanzer KE, Roos J, Kelly RB, Davis GW. Dap160/intersectin scaffolds the periactive zone to achieve high-fidelity endocytosis and normal synaptic growth. Neuron (2004) 43(2):207-19. doi:10.1016/j.neuron.2004.07.001

18. Chang KT, Min KT. Upregulation of three Drosophila homologs of human chromosome 21 genes alters synaptic function: implications for down syndrome. Proc Natl Acad Sci U S A (2009) 106(40):17117-22. doi:10.1073/pnas.0904397106

19. Evergren E, Gad H, Walther K, Sundborger A, Tomilin N, Shupliakov O. Intersectin is a negative regulator of dynamin recruitment to the synaptic endocytic zone in the central synapse. J Neurosci (2007) 27(2):379-90. doi:10.1523/ JNEUROSCI.4683-06.2007

20. Pechstein A, Bacetic J, Vahedi-Faridi A, Gromova K, Sundborger A, Tomlin $\mathrm{N}$, et al. Regulation of synaptic vesicle recycling by complex formation between intersectin 1 and the clathrin adaptor complex AP2. Proc Natl Acad Sci U S A (2010) 107(9):4206-11. doi:10.1073/pnas. 0911073107

21. Henne WM, Boucrot E, Meinecke M, Evergren E, Vallis Y, Mittal $\mathrm{R}$, et al. FCHo proteins are nucleators of clathrinmediated endocytosis. Science (2010) 328(5983):1281-4. doi:10.1126/science.1188462

22. Khanna R, Li Q, Stanley EF. "Fractional recovery" analysis of a presynaptic synaptotagmin 1-anchored endocytic protein complex. PLoS ONE (2006) 1:e67. doi:10.1371/

23. Yu Y, Chu PY, Bowser DN, Keating DJ, Dubach D, Harper I, et al. Mice deficient for the chromosome 21 ortholog Itsn 1 exhibit vesicletrafficking abnormalities. Hum Mol Genet (2008) 17(21):3281-90. doi: $10.1093 / \mathrm{hmg} / \mathrm{ddn} 224$

24. Thomas S, Ritter B, Verbich D, Sanson C, Bourbonnière L, McKinney RA, et al. Intersectin regulates dendritic spine development and somatodendritic endocytosis but not synaptic vesicle recycling in hippocampal neurons. J Biol Chem (2009) 284(18):12410-9. doi: 10.1074/jbc.M809746200

25. Sakaba T, Kononenko NL, Bacetic J, Pechstein A, Schmoranzer J, Yao L, et al. Fast neurotransmitter release regulated by the endocytic scaffold intersectin. Proc Natl Acad Sci U S A (2013) 110(20):8266-71. doi:10. 1073/pnas.1219234110

26. Nishimura T, Yamaguchi T, Tokunaga A, Hara A, Hamaguchi T, Kato $\mathrm{K}$, et al. Role of numb in dendritic spine development with a $\mathrm{Cdc} 42$ GEF intersectin and EphB2. Mol Biol Cell (2006) 17(3):1273-85. doi: 10.1091/mbc.E05-07-0700 journal.pone.0000067 
27. Sengar AS, Ellegood J, Yiu AP, Wang H, Wang W, Juneja SC, et al. Vertebrate intersectinl is repurposed to facilitate cortical midline connectivity and higher order cognition. J Neurosci (2013) 33(9):405565. doi:10.1523/JNEUROSCI.442812.2013

28. Ory S, Gasman S. Rho GTPases and exocytosis: what are the molecular links? Semin Cell Dev Biol (2011) 22(1):27-32. doi:10.1016/j.semcdb. 2010.12.002

29. Momboisse F, Houy S, Ory S, Calco V, Bader MF, Gasman S. How important are Rho GTPases in neurosecretion? J Neurochem (2011) 117(4):623-31. doi:10.1111/j.14714159.2011.07241.x

30. Gasman S, Chasserot-Golaz S, Malacombe M, Way M, Bader MF. Regulated exocytosis in neuroendocrine cells: a role for subplasmalemmal $\quad \mathrm{Cdc} 42 / \mathrm{N}$ WASP-induced actin filaments. Mol Biol Cell (2004) 15(2):520-31. doi:10.1091/mbc.E03-06-0402

31. Malacombe M, Ceridono M, Calco V, Chasserot-Golaz S, McPherson
PS, Bader MF, et al. Intersectin1L nucleotide exchange factor regulates secretory granule exocytosis by activating $\mathrm{Cdc} 42$. $E M B O$ $J$ (2006) 25(15):3494-503. doi:10. 1038/sj.emboj.7601247

32. Momboisse F, Ory S, Calco V, Malacombe M, Bader MF, Gasman S. Calcium-regulated exocytosis in neuroendocrine cells: intersectin1L stimulates actin polymerization and exocytosis by activating Cdc42. Ann N Y Acad Sci (2009) 1152:209-14. doi:10.1111/j. 1749-6632.2008.03998.x

33. Barg S, Machado JD. Compensatory endocytosis in chromaffin cells. Acta Physiol (Oxf) (2008) 192(2):195201. doi:10.1111/j.1748-1716.2007. 01813.x

34. Khanna R, Li Q, Schlichter LC, Stanley EF. The transmitter release-site CaV2.2 channel cluster is linked to an endocytosis coat protein complex. Eur J Neurosci (2007) 26(3):560-74. doi:10.1111/j.14609568.2007.05681.x

35. Zhai RG, Bellen HJ. The architecture of the active zone in the presynaptic nerve terminal. Physiology (Bethesda) (2004) 19:262-70. doi:10.1152/physiol.00014.2004

36. Haucke V, Neher E, Sigrist SJ. Protein scaffolds in the coupling of synaptic exocytosis and endocytosis. Nat Rev Neurosci (2011) 12(3):127-38. doi:10.1038/nrn2948 37. Winther AM, Jiao W, Vorontsova O, Rees KA, Koh TW, Sopova E, et al. The dynamin-binding domains of Dap160/intersectin affect bulk membrane retrieval in synapses. J Cell Sci (2013) 126(Pt 4):1021-31. doi:10.1242/jcs. 118968

38. Ceridono M, Ory S, Momboisse F, Chasserot-Golaz S, Houy S, Calco $\mathrm{V}$, et al. Selective recapture of secretory granule components after full collapse exocytosis in neuroendocrine chromaffin cells. Traffic (2011) 12(1):72-88. doi:10.1111/ j.1600-0854.2010.01125.x

Conflict of Interest Statement: The authors declare that the research was conducted in the absence of any commercial or financial relationships that could be construed as a potential conflict of interest.

Received: 01 July 2013; accepted: 09 August 2013; published online: 27 August 2013.

Citation: Gubar O, Morderer D, Tsyba L, Croisé P, Houy S, Ory S, Gasman S and Rynditch A (2013) Intersectin: the crossroad between vesicle exocytosis and endocytosis. Front. Endocrinol. 4:109. doi: 10.3389/fendo.2013.00109

This article was submitted to Neuroendocrine Science, a section of the journal Frontiers in Endocrinology.

Copyright (c) 2013 Gubar, Morderer, Tsyba, Croisé, Houy, Ory, Gasman and Rynditch. This is an open-access article distributed under the terms of the Creative Commons Attribution License (CC $B Y)$. The use, distribution or reproduction in other forums is permitted, provided the original author(s) or licensor are credited and that the original publication in this journal is cited, in accordance with accepted academic practice. No use, distribution or reproduction is permitted which does not comply with these terms. 